\title{
Electrospun plant-derived natural biomaterials for Tissue engineering
}

\author{
Jayarama Reddy Venugopal ${ }^{\varpi}$, Sreepathy Sridhar and Seeram Ramakrishna
}

\begin{abstract}
Plant-derived natural products are being used in medicine, and they are easily available for the production and use in tissue engineering based biological applications. Utilization of plant materials to treat human diseases is a common practice followed over many decades. In fact plant and its derivatives have been actively included in health management over thousands of years. The advent of phytochemical and phytopharmacological sciences has opened an arena to elucidate the structural and biological composition of several medicinal plant products. Their pharmacological effects depend on the supply of highly active water soluble compounds; however, due to their large molecular size most compounds are unable to cross the lipid membranes of the cells and therefore result in poor absorption resulting in loss of bioavailability and efficacy. Electrospinning makes it possible to combine the advantages of utilizing these plant materials in the form of nanofibrous scaffolds for delivering the active constituent at a sufficient concentration during the entire treatment period to the host site. The aim of this review is to highlight the potential applications of electrospun nanofibrous scaffolds based systems and herbal medicines in tissue engineering.
\end{abstract}

Keywords: electrospinning; nanofibers; Aloe vera; xylan; Cissus quadrangularis

Received: 26 May 2014 / Accepted revised version: 9 July 2014 / Published online: 15 August 2014

(C) Horizon e-Publishing Group

CITATION

Venugopal, J., Sreepathy, S., \& Ramakrishna, S. (2014). Electrospun plant-derived natural biomaterials for Tissue engineering. Plant Science Today, 1(3), 151-154. http://dx.doi.org/10.14719/pst.2014.1.3.65

\section{AUTHORS' AFFILIATION}

Centre for Nanofibers and Nanotechnology, NUSNNI, Faculty of Engineering, National University of Singapore, Singapore 117576

CORRESPONDENCE

凶J. Venugopal, Phone: 65 - 65164272 Fax: 65 - 68725563 E-mail: nnijrv@nus.edu.sg

\section{Introduction}

Tissue engineering and nanotechnology have advanced a general strategy combining the cellular elements of living tissue with sophisticated functional biocomposites to produce living structures of sufficient size and function at a low cost for clinical significance (Venugopal, Molamma, Shayanti, Rajeswari, \& Ramakrishna, 2012). In the last decade there has been an explosion of advances in the field of nanotechnology and tissue engineering offering the niche for regenerating most organs of the human body. The diversity of chemical structures, conjointly with precise control of the molecular architecture and morphology, provides an insight into numerous ways of using polymers in high-technology and biological applications. Therefore, these nanofibrous materials are prime candidates for many momentous applications such as reinforcement in nanocomposites, tissue engineering scaffolds and filtration devices. Despite the synthesis of distinct polymers, lacking a functional domain to interface with biological systems poses hindrance to tissue engineering. Natural polymers too have their cynical features like lack of control of molecular weight, chain configuration, and polymerization kinetics, versatility of chemistry but have advantages over synthetic polymers, such as low cost, biocompatibility, nontoxicity, and biodegradability. Hence the need for hybrid polymers came to existence while making their molecular chains look more or less similar to biological proteins. The natural products are expected to play significant roles in creating new and better chemopreventive and therapeutic agents. The convenience of using synthetic polymers along with these natural polymers is that its properties can be tuned easily to favor cell adhesion and proliferation in tissue engineering. Most of the natural biopolymers are electrospun using organic solvents which may prove toxic for tissue engineering applications. As plants are a source of many bioactive compounds and many plant ingredients are traditionally used to accelerate healing, scientists go back to traditional folk medicines as they are generally characterized by high acceptability and tolerance. The healing potential of phytomedicines is often associated 
with angiogenesis, which is a critical step of wound healing. Mimicking the porous topography of natural extracellular matrix (ECM) is advantageous for successful regeneration of damaged tissues or organs. Nanotechnology being one of the most promising and growing technology has huge potential in the field of tissue engineering (Venugopal et al., 2012; Venugopal, Shayanti, Rajeswari, Sridhar, \& Ramakrishna, 2013a; Venugopal, Sridhar, Rajeswari, Shayanti, Balamurugan, \& or organ in vitro or in vivo. Several methods are available for the preparation of an ideal scaffolds but the electrospinning process has attracted a great deal of attention due to its cost effective and easiest way to produce ultrafine fibers from polymer solutions with diameters in the range of nanometre to sub-micrometers that exhibit high surface-area-to-volume ratio using electrostatic forces (Venugopal et al., 2013a; 2013 ${ }^{\text {b }}$ ). The topological structure of electrospun products can mimic
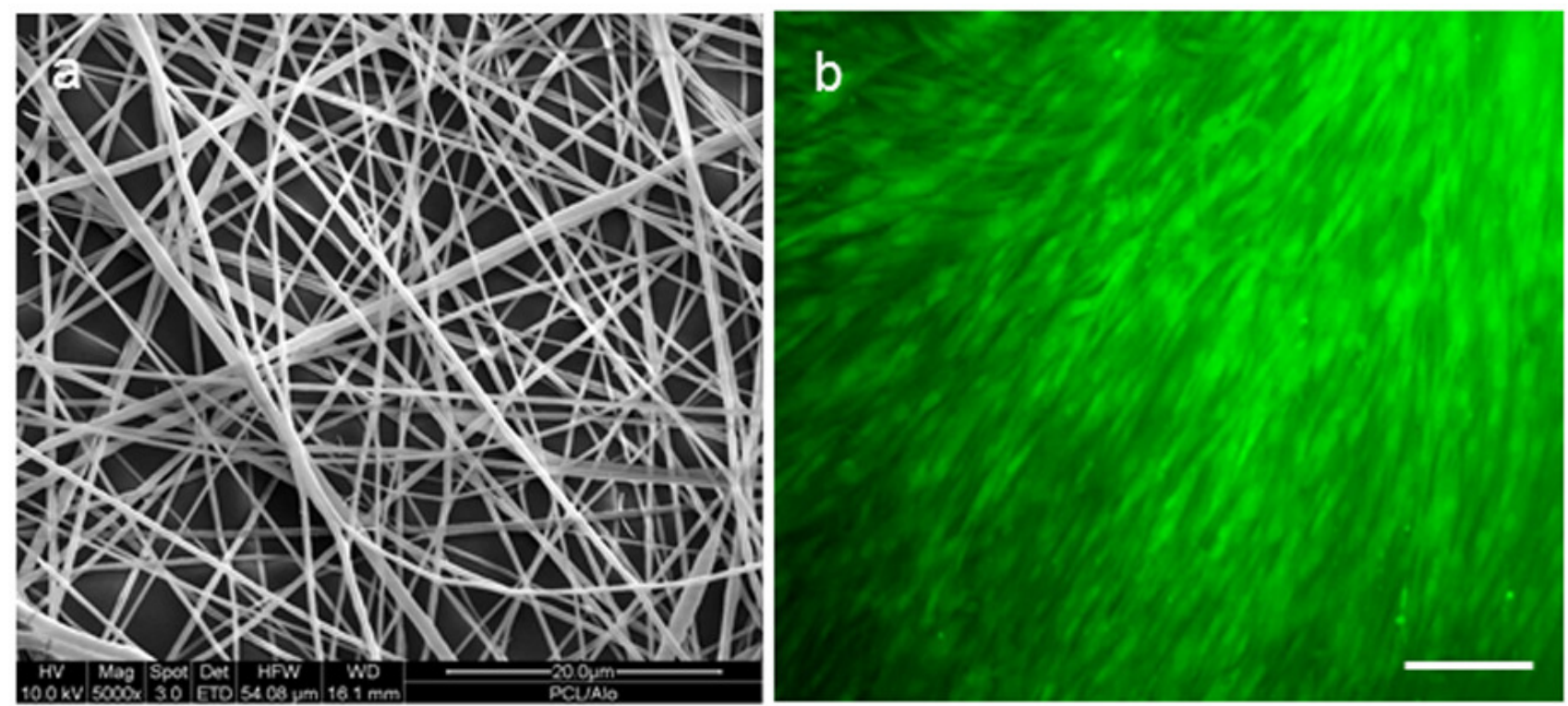

Figure 1. Morphology of electrospun PCL/Aloe vera nanofibers (352 $\pm 75 \mathrm{~nm})$ (a) and 5-

chloromethylfluorescein diacetate (CMFDA) dye expression in fibroblast cultured on PCL/A. vera nanofibers for skin tissue engineering (b), (NUSNNI).

Ramakrishna, $2013^{\mathrm{b}}$ ). It plays an essential role in successful tissue repair as it provides sustained nutrient supply to enable cell proliferation. It therefore functions as an intact delivery system and also facilitates the clearance of debris. This review in particular highlights the potentials of natural compounds as substrate for tissue engineering applications.

\section{Aloe vera (L.) Burm.f.}

It is one of the medicinal herbs belonging to the Liliaceae family. A. vera has been used in the treatment of a variety of disorders including infections, dermatologic conditions and used as a laxative since ancient times. The gel inside the leaf consists of 99\% water with long chain polysaccharide of acetylated glucomannan and other carbohydrates. It also contains the complex of amino acids, salicylic acid, ascorbic acid, vitamin $A$ and $E$ that is attributed to its anti-oxidant properties. Aloe gel prevents skin dryness due to the high volume of water. Tissue engineering has emerged as a promising approach to overcome the wound healing drawbacks with the use of scaffolds that acts as a drug carrier as well mimics natural extracellular matrix. Scaffolds are highly porous, typically macromolecular, solids that are used to synthesize tissue the ECM and enhance both cell migration and proliferation in tissue engineering. Nanotopographical features such as pores, ridges, grooves, fibers, nodes or a combination of these have been reported to influence the cell behaviour. Possibility to immobilize antibiotics, enzymes, antimicrobial peptides and growth hormones to nanofibers, or encapsulation into fiber matrices opens a new field in biomedical engineering. We have successfully fabricated the polycaprolactone/A. vera nanofibrous scaffolds using electrospinning, upon which human dermal fibroblasts (ATCC, US) were cultured in an attempt to develop dermal substitute for wound healing and skin tissue engineering (Fig. 1). Cytoskeletal organization of the nanofibers was related to cell adhesion, spreading functions on the substrates. Suganya, Venugopal, Ramakrishna, Lakshmi, \& Giri Dev (2014a) proved that the fibroblasts can have better and faster attachment, proliferation and guided growth on the polycaprolactone/A. vera (PCL/AV) nanofibers for wound healing and skin tissue engineering.

Recently, A. vera has been proven to accelerate the healing of open wounds in type 2 diabetic radiation-exposed rats when administered orally. A. vera, 
because of its wound healing and anticancer property, has been used together with nanofibers for better delivery to the cells. Porous nanofibrous scaffolds provide a common and versatile platform for skin tissue engineering. Though there is a tangible progress in skin tissue engineering with these porous structures, the aspect of controlled release of drugs, growth factors and oxygen release which are crucial contributors for wound healing cascade remain much unexplored. Fabrication of the porous biodegradable polymers with $A$. vera fibrous membranes has good interest not only for their renewable character and nontoxic properties but also offer biocompatibility and biodegradability. Generally plant hydrogels possess a degree of flexibility due to their significant water content and they are potential substrates for tissue engineering, controlled drug release, agriculture and hygiene products. Most of the polymers are electrospun using organic solvents either single solvent system or mixture of solvents depending on the solubility of polymeric materials. Electrospinning technology has tremendous
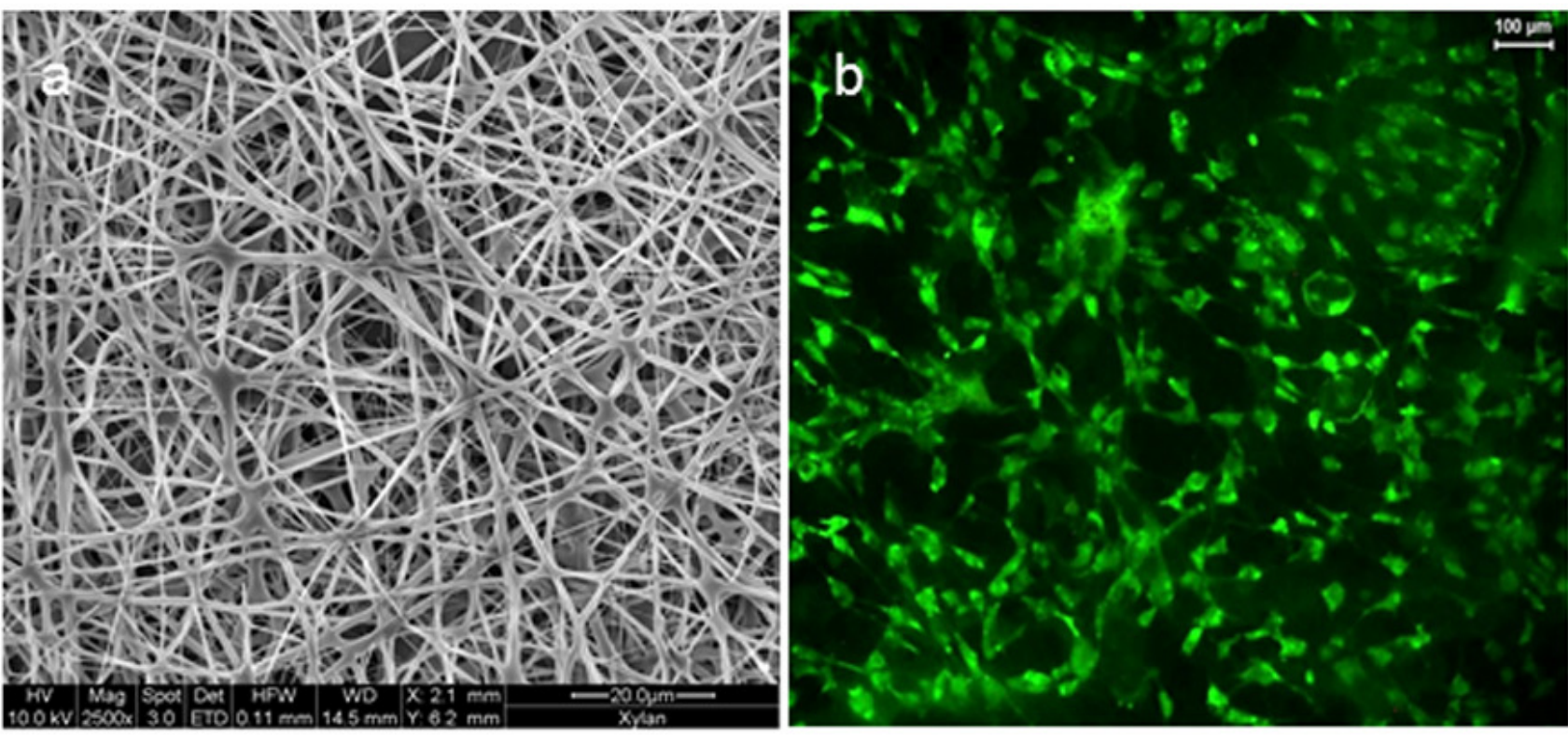

Figure 2. Morphology of electrospun PVA/Xylan nanofibers (665 $\pm 93 \mathrm{~nm}$ ) (a) CMFDA dye expression in cardiomyocytes cultured on PVA/Xylan nanofibers for cardiac tissue engineering (b), (NUSNNI).

potential for wound healing applications (Kimberley et al., 2014). Due to the inherent wound healing property of $A$. vera, it has been used as nanofibrous scaffold which mimics the natural extracellular matrix to host the cells for the skin and bone tissue regeneration. It has been widely used to mix with PCL/AV nanofibrous scaffolds for its wound healing property and also engineering bone tissues along with nanohydroxyapatite (HA) for bone tissue regeneration (Suganya, Venugopal, Ramakrishna, Lakshmi, \& Giri Dev, 2014 ).

\section{Xylan}

Natural products are major resource for most of the active ingredients of medicines (Morris, 2011). Therapeutic properties and medicinal benefits of natural products can be linked to the presence of a wide array of bioactives especially alkaloid, diterpenoid, triterpenoid, polysaccharide, and polyphenols. It is generally believed that natural products have enormous yet unexploited potential and advantages of natural product derived molecules as candidates for drug development are widely described. Naturally available xylan based hydrogels are of potential to fabricate nonwoven nanofibrous scaffolds from biological and synthetic polymers for tissue engineering applications (Krishnan et al., 2012). Synthetic polymers are specifically engineered (both physically and chemically) to aid tissue regeneration and it is known that the surface microstructure and chemistry of these engineered substrates can influence the ability of cells and tissues to attach, grow and function normally. Natural collagen scaffolds works well for wound healing but processing and sterilization are essential to control infections for patients. Moreover, most of the natural biopolymers are electrospun using organic solvents which may prove toxic for tissue engineering applications. Fabricated scaffolds may contain small amount of organic solvent that could affect the cell growth and proliferation. Electrospinning of biocompatible polymers using aqueous solvents will help to overcome this problem and are therefore highly desirable for cell culture and tissue engineering. Xylan is an abundant biopolymer in the plant kingdom and generally used in pharmaceutical industry for developing drug delivery systems. Hemicelluloses are polysaccharides that occur together with cellulose in most 
plant tissues. The predominant hemicellulose present in hardwoods such as birch and aspen is an 0-(4-0-methylglucurono) xylan and these are abundant in naturally occurring polysaccharides representing a large and cost-effective source of polymeric materials in tissue engineering applications.

Worldwide, there is growing pressure to introduce an enforceable definition that could be used to regulate the use, approval and possible labelling of nanotechnology products. We have earlier reported the use of electrospun nanofibrous scaffolds of Xylan polysaccharide and polyvinyl alcohol (PVA) for cardiac tissue engineering applications (Venugopal et al., 2013a). The fiber diameters of Xylan/PVA nanofibers are around $665 \pm 93 \mathrm{~nm}$ have better mechanical properties, suitable swelling properties to enable culture of cardiomyocytes that could then be used as a cardiac patch for the regeneration of infarcted myocardium (Fig. 2).

\section{Cissus quadrangularis $\mathrm{L}$.}

It is a medicinal plant possessing osteogenic activity and are attaining increasing interest as a potential therapeutic agent for enhancing bone healing. The extracts of these plants are reported to contain phytoestrogenic steroids, ascorbic acid, flavonoids, carotene and calcium. Petroleum ether extract of $C$. quadrangularis (CQ) rich in phytosterol enhances bone marrow mesenchymal stem cell proliferation and foetal bone growth. Suganya, Venugopal, Ramakrishna, Lakshmi, \& Giri Dev (2014c) reported the use of herbal scaffolding approach for bone tissue engineering applications and had successfully fabricated the PCL-CQ-HA nanofibrous scaffolds for bone tissue engineering. The herbal based nanofibrous scaffolds proved that the PCL-CQ and PCL-CQ-HA enhanced osteoblast proliferation and mineralization however PCL-CQ-HA responses to cellular interaction was more pronounced and therefore CQ in combination with HA will be a promising approach for solving the current issues related to bone defects in bone tissue engineering.

\section{Conclusion}

Electrospinning being recognized as a simple technique for the fabrication of polymer nanofibers has produced nanofibers with several astonishing characteristics such as very large surface area-to-volume ratio, surface function flexibilities and superior mechanical performance (e.g. stiffness and tensile strength) compared to any other known form of the materials. The production and designing of effective antimicrobial materials has become a highly desired objective in maintaining primary health care systems. Many plant-derived biomaterials are beginning to emerge opening up a new frontier on phytochemcial based tissue engineering applications. These biomaterials need to be screened for the pharmacological effects of the various active constituents present in the plant materials such as phytosteroids, anthraquinones, etc. and those with beneficial properties will eventually make its advent into market. The nanofibrous scaffolds which have such desired phyto-chemical property would be considered to be the ideal candidate for drug delivery and tissue engineering.

\section{Acknowledgement}

This study was supported by the NRF-Technion (R-398-001-065-592) and NUSNNI, National University of Singapore, Singapore.

\section{References}

Kimberley, T., Suganya, C., Venugopal, J., Biswas, A., Mahesh, C., Ramakrishna, S., ... \& Fong, C. A. (2014). Nanoscaffold Impregnated With Human Wharton's Jelly Stem Cells or Its Secretions Improves Healing of Wounds. Journal of Cellular Biochemistry, 115(4), 794-803. PMid:24265214 http://dx.doi.org/10.1002/jcb.24723

Krishnan, R., Rajeswari, R., Venugopal, J., Sridhar, R., Shayanti, M., \& Ramakrishna, S. (2012). Polysaccharide nanofibrous scaffolds as a model for in vitro skin tissue regeneration. Journal of Materials Science: Materials in Medicine, 23, 1511-1519. PMid:22491895 http://dx.doi.org/10.1007/s10856-012-4630-6

Morris, V. J. (2011). Emerging roles of engineered nanomaterials in the food industry. Trends in Biotechnology, 29, 509-516. http://dx.doi.org/10.1016/j.tibtech.2011.04.010 PMid:21664709

Suganya, S., Venugopal, J., Ramakrishna, S., Lakshmi, B., \& Giri Dev, V. R. (2014)a. Aloe vera incorporated biomimetic nanofibrous scaffold: a regenerative approach for skin tissue engineering. Iran Polymer Journal, 23(3), 237-248. http://dx.doi.org/10.1007/s13726-013-0219-2

Suganya, S., Venugopal, J., Ramakrishna, S., Lakshmi, B., \& Giri Dev, V. R. (2014)b. Herbally Derived Polymeric Nanofibrous Scaffolds for Bone Tissue Regeneration. Journal of Applied Polymer Science, 131, 9835 (1-11). http://dx.doi.org/10.1002/app.39835

Suganya, S., Venugopal, J., Ramakrishna, S., Lakshmi, B., \& Giri Dev, V. R. (2014)c. Aloe vera/Silk Fibroin/Hydroxyapatite Incorporated Electrospun Nanofibrous Scaffold for Enhanced Osteogenesis. Journal of Biomaterials Applications, 4(1), 9-19. http://dx.doi.org/10.1177/0885328213513934

Venugopal, J., Molamma, P., Shayanti, M., Rajeswari, R., \& Ramakrishna, S. (2012). Biomaterial strategies for alleviation of Myocardial Infarction. Journal of Royal Society Interface, 9, 1-19. http://dx.doi.org/10.1098/rsif.2011.0301 PMid: 21900319

Venugopal, J., Shayanti, M., Rajeswari, R., Sridhar, R., \& Ramakrishna, S. (2013)a. Xylan polysaccharides fabricated into nanofibrous substrate for Myocardial Infarction. Materials Science and Engineering C, 33, 1325-1331. PMid:23827578 http://dx.doi.org/10.1016/j.msec.2012.12.032

Venugopal, J., Sridhar, R., Rajeswari, R., Shayanti, M., Balamurugan, R., \& Ramakrishna S. (2013) ${ }^{\text {b }}$ Nanofibrous structured Biomimetic Strategies for Skin Tissue Regeneration. Wound Repair and Regeneration, 21, 1-16. PMid:23126632. http://dx.doi.org/10.1111/j.1524-475X.2012.00861.x 